\title{
INVARIANTS OF FORMAL GROUP LAW ACTIONS ${ }^{1}$
}

\author{
BY ROBERT M. FOSSUM
}

0. Introduction. In this note, $k$ denotes a field of characteristic $p>0$, and the letters $T, X$ and $Y$ are formal indeterminants. Let $F: k[[T]] \rightarrow k[[X, Y]]$ be a (fixed) one-dimensional formal group law [Dieudonné, Hazewinkel, Lazard, Lubin] of height $h \geq 0$. Let $V$ denote a $k[[T]]$ module of finite length. Suppose $\operatorname{Ann}(V)=\left(T^{n}\right)$. Let $q=p^{e}$ denote the least power of $p$ such that $n \leq q$. It follows that the symmetric powers $S_{r}(V)$ over $k$ become $k[[T]]$-modules, annihilated by $T^{q}$, through the formal group law, viz: If $F(T)=X+Y+$ $\sum_{i, j \geq 1} C_{i j} X^{i} Y^{j}$ and $f$ is in $S_{t}(V)$ and $g$ is in $S_{s}(V)$, then

$$
T(f g)=f T g+(T f) g+\sum C_{i j}\left(T^{i} f\right)\left(T^{j} g\right)
$$

in $S_{t+s}(V)$.

Denote by $S .(V)$ the symmetric algebraic on $V$; so

$$
S .(V):=\bigoplus_{r>0} S_{r}(V)
$$

Then $S .(V)$ is a $k[[T]]$-module annihilated by $T^{q}$. The main purpose of this note is to announce and outline a proof of the theorem below. Several consequences and examples are included.

Theorem. Let $S .(V)^{F}:=\{f \in S .(V): T f=0\}$. The set $S .(V)^{F}$ is a normal noetherian subring of $S .(V)$ of the same Krull dimension. Furthermore, $S .(V)^{F}$ is factorial.

1. An outline of the proof. To prove the Theorem one can consider two cases: ht $F=h=1$ and ht $F=h \neq 1$. In case ht $F=1$, the action on $S .(V)$ is equivalent to an action of the cyclic group $\mathbf{Z} / q Z$ on $S .(V)$. This case is considered, in full generality, in [Fossum, Griffith] and [Almkvist, Fossum].

So consider the case ht $F \neq 1$. It can be shown that there is a fixed power $s$ of $p$, depending only on ht $F$, such that $S .(V)^{s} \subset S .(V)^{F}$. Then one can extend the action of $T$ to the field of fractions $L$ of $S .(V)$ via

$$
T(f / g)=T\left(f g^{s-1}\right) / g^{s} \text {. }
$$

Then one concludes that $L^{F}$ is a field and

$$
S .(V)^{F}=L^{F} \cap S .(V),
$$

which shows that $S .(V)^{F}$ is a Krull domain and $S .(V)^{F} \supset k\left[S .(V)^{s}\right]$, which shows that $S .(V)^{F}$ is noetherian and $S .(V)$ is integral over $S .(V)^{F}$. Hence,

Received by the editors May 2, 1983.

1980 Mathematics Subject Classification. Primary 14L05, 14L30, 13H10, 13F15.

${ }^{1}$ This research has been supported by the National Science Foundation.

(C) 1983 American Mathematical Society $0273-0979 / 82 \$ 1.00+\$ .25$ per page 
the extension $S .(V)^{F} \rightarrow S .(V)$ is (PDE) (cf. [Fossum]). It remains to prove that $S .(V)^{F}$ is factorial. As $S .(V)$ and $S .(V)^{F}$ are graded, it is sufficient to consider homogeneous ideals and to show that each homogeneous, prime, divisorial ideal $\mathfrak{p}$ in $S .(V)^{F}$ is principal. One could accomplish this by using results in [Waterhouse], but there is also a straightforward calculation.

Suppose $\mathfrak{p}$ is a homogeneous prime divisorial ideal in $S .(V)^{F}$. Let $(\mathfrak{p} S .(V))^{* *}$ denote the divisorial ideal it generates in $S .(V)$. This ideal is principal, generated by a homogeneous element $f$ in $S .(V)$. It follows that

$$
f^{-1} S .(V)=\{x \in L: x \mathfrak{p} \subset S .(V)\} .
$$

Since $f^{-1} \mathfrak{p} \subset S .(V)$ and $T(\mathfrak{p})=0$, it follows easily that $T\left(f^{-1}\right) \mathfrak{p} \subset S .(V)$. Hence, there is an element $a \in S .(V)$ such that

$$
T\left(f^{-1}\right)=f^{-1} a, \text { or } T\left(f^{s-1}\right)=f^{s-1} a .
$$

Since degree $T\left(f^{s-1}\right)=$ degree $f^{s-1}$, it follows that degree $a=0$, or that $a \in k$. Hence $T(a)=0$. Then

$$
T^{r}\left(f^{s-1}\right)=f^{s-1} a^{r} .
$$

Since $T^{q}=0$, one obtains that $a^{q}=0$ or $a=0$. Hence, $T\left(f^{s-1}\right)=0$. Then

$$
0=T\left(f^{s}\right)=f T\left(f^{s-1}\right)+T(f) f^{s-1}=T(f) f^{s-1} \text {. }
$$

Hence, $T(f)=0$, so $f \in S .(V)^{F}$. From this one concludes that $\mathfrak{p}=f S .(V)^{F}$. This concludes the (outline) of the proof. Details that are omitted will appear elsewhere.

2. Examples. For each $n \in \mathbf{N}$, set $V_{n}:=k[[T]] /\left(T^{n}\right)$. The Hilbert series of $S .\left(V_{n}\right)^{F}$ is, by definition, the power series

$$
\sum_{r=0}^{\infty}\left(\operatorname{rk}_{k} S_{r}\left(V_{n}\right)^{F}\right) t^{r} \in \mathbf{Z}[[t]]
$$

Denote this series by $H_{t}\left(V_{n}, F\right)$. (The dependence on $p$ is implicit.) In case $F(T)=X+Y+X Y$ (and so ht $F=1$ ) these Hilbert series have been studied extensively in [Almkvist, Fossum]. In particular, for $n=q=p^{e}$,

$$
H_{t}\left(V_{p^{e}}, F\right)=p^{-e}\left\{(1-t)^{-p^{e}}+\sum_{j=1}^{e}\left(p^{j}-p^{j-1}\right)\left(1-t^{p^{j}}\right)^{-p^{e-j}}\right\} .
$$

Some results are now available for the additive formal group law $F(T)=X+Y$ (so ht $F=0$ ). In this case, for $q=p^{e}$,

$$
\begin{aligned}
H_{t}\left(V_{q}, X+Y\right) & =q^{-1}\left\{(1-t)^{-q}+(q-1)\left(1-t^{p}\right)^{-q / p}\right\} \\
& =\left(1-t^{p}\right)^{-q / p}+q^{-1}\left\{(1-t)^{-q}-\left(1-t^{p}\right)^{-q / p}\right\} .
\end{aligned}
$$

This can be used to show that

$$
H_{t}\left(V_{q-1}, X+Y\right)=\left(1-t^{p}\right)^{-q / p}+(1-t) q^{-1}\left\{(1-t)^{-q}-\left(1-t^{p}\right)^{-q / p}\right\} .
$$

This rational function of $t$ is not unimodal for most $q$, and hence $S .\left(V_{q-1}\right)^{(X+Y)}$ is not Gorenstein for these $q$. Thus $S .\left(V_{q-1}\right)^{(X+Y)}$ is not Cohen-Macaulay. Evidence suggests that $S .\left(V_{n}\right)^{F}$ is not Cohen-Macaulay for $n \geq 4$, except in 
case $p=2$ and $n=4$ (cf. [Stanley]). The case $p=2$ has been studied by [Bertin]. The Hilbert series

$$
H_{t}\left(V_{4}, X+Y+X Y\right)=\frac{1-t+t^{2}+t^{3}}{(1-t)^{2}\left(1-t^{2}\right)^{2}\left(1+t^{2}\right)},
$$

which shows that $S .\left(V_{4}\right)^{X+Y+X Y}$ is not Cohen-Macaulay, since it is factorial and not Gorenstein.

The Hilbert series, $p=2$, for $V_{4}$ is

$$
H_{t}\left(V_{4}, X+Y\right)=\frac{\left(1+t^{3}\right)}{(1-t)\left(1-t^{2}\right)^{3}}
$$

A calculation shows, for $S .\left(V_{4}\right)=k\left[X_{3}, X_{2}, X_{1}, X_{0}\right]$ with $T X_{3}=X_{2}, T X_{2}=$ $X_{1}, T X_{1}=X_{0}, T X_{0}=0$, and $T(f g)=f(T g)+(T f) g$, that

$$
S .\left(V_{4}\right)^{X+Y}=k\left[X_{0}, X_{1}^{2}, X_{2}^{2}, X_{3}^{2}, X_{3} X_{0}^{2}+X_{2} X_{1} X_{0}+X_{1}^{3}\right]
$$

which is a complete intersection.

Further results will appear in more detail.

\section{REFERENCES}

[G. Almkvist and R. Fossum] Decomposition of exterior and symmetric powers of indecomposable $\mathbf{Z} / p \mathbf{Z}$-modules in characteristic $p$ and relations to invariants, Sém. d'Algèbre Paul Dubreil, M.-P. Malliavin, Lecture Notes in Math., No. 641, Springer-Verlag, Berlin and New York, 1978, pp. 1-111.

[M.-J. Bertin] Anneaux d'invariants d'anneaux de polynômes, en characteristique p, C. R. Acad. Sci. Paris 264 (1967), 653-656.

[Jean Dieudonné] Introdution to the theory of formal groups, Dekker, New York, 1973.

[Robert Fossum] The divisor class group of a Krull domain, Ergeb. Math. Grenzgeb., Bd. 74, Springer-Verlag, Berlin and New York, 1973.

[R. Fossum and P. A. Griffith] Complete local factorial rings which are not Cohen-Macaulay in characteristic p, Ann. Sci. École Norm. Sup. (4) 8 (1975), 189-200.

[Michael Hasewinkel] Formal groups and applications, Academic Press, New York, 1978.

[Jonathan Lubin] Introduction à la theorie des groupes formels, Secretariat Math. École Norm. Sup. Paris, 1970.

[Michel Lasard] Commutative formal groups, Lecture Notes in Math., No. 443, SpringerVerlag, Berlin and New York, 1975.

[Richard Stanley] Hilbert functions of graded algebras, Adv. Math. 28 (1978), 57-83.

[William Waterhouse] Divisor classes in pseudo-Galois extensions, Pacific J. Math. 36 (1971), 541-548.

Institut for Algebraic Meditation and Department of Mathematics, UniVERSITY OF ILLINOIS, URBANA, ILLINOIS 61801 
